\title{
Dynamics of a Heroin Epidemic Model with Very Population
}

\author{
Xiaoyan Wang ${ }^{1}$, Junyuan Yang ${ }^{1}$, Xuezhi $\mathbf{L i}^{2}$ \\ ${ }^{1}$ Department of Applied Mathematics, Yuncheng University, Yuncheng, China \\ ${ }^{2}$ Department of Mathematics, Xinyang Normal University, Xinyang, China \\ E-mail: yangjunyuan00@126.com \\ Received March 26, 2011; revised April 16, 2011; accepted April 19, 2011
}

\begin{abstract}
Based on the model provided by the Mulone and Straughan [1], we relax the population which are constant and obtain the drug-free equilibrium which is global asymptotically stable under some conditions. The system has only uniqueness positive endemic equilibrium which is globally asymptotically stable by using the second compound matrix.
\end{abstract}

Keywords: Heroin Epidemic Model, Equilibrium, Global Stability

\section{Introduction}

Heroin is an opiate drug that is synthesized from morphine, a naturally occurring substance extracted from the seed pod of the Asian opium poppy plant. Heroin usually appears as a white or brown powder or as a black sticky substance, known as "black tar heroin" [2]. Heroin users are at high risk for addiction - it is estimated that about 23 percent of individuals who use heroin become dependent on it. The spread of heroin habituation and addiction presents many of the well-known phenomena of epidemics, including rapid diffusion and clear geographic boundaries. It is unrealistic to repeat the experiment on the human body for obtaining the statistic data. Mathematical models play very important role in dealing with these problems. Mathematical modellings are very useful tools to predict how classes of drug takers behave, and provide a good suggestion for the treatment strategies. It is interest to explore the problem mathematically, reducing the factors to the essential transmission mechanism, in real life, is much more complex because of imponderable and often intervening biological, psychological, and social conditions.

During recent years, many mathematical models have been developed to describe the Heroin epidemic model (see $[1,3])$. One of the recent model divided the mathematical problem into tree class, namely susceptibles, heroin users or alcoholics, and heroin users or alcoholics undergoing treatment which denote by $S(t), U_{1}(t)$ and $U_{2}(t)$, respectively. The model is

$$
\begin{aligned}
& \frac{\mathrm{d} S}{\mathrm{~d} t}=\Lambda-\frac{\beta_{1} S U_{1}}{N}-\mu S, \\
& \frac{\mathrm{d} U_{1}}{\mathrm{~d} t}=\frac{\beta_{1} S U_{1}}{N}+\frac{\beta_{3} U_{1} U_{2}}{N}-\left(p+\mu+\delta_{1}\right) U_{1}, \\
& \frac{\mathrm{d} U_{2}}{\mathrm{~d} t}=p U_{1}-\frac{\beta_{3} U_{1} U_{2}}{N}-\left(\mu+\delta_{2}\right) U_{2}
\end{aligned}
$$

where the number of individuals enter the susceptible population at a rate, $\Lambda$, die at rate $\mu$. Infection of a drug user occurs through a simple mass action process $\beta_{1} S U_{1} / N$, where $\beta_{1}$ is probability of becoming a drug user, per unit time. The probability of a drug user in treatment relapsing to untreated use, per unit time is $p$. Infected heroin users die at enhanced rate $\delta_{1}$, treatment infected heroin users die at enhanced rate $\delta_{2}$. In this model, the authors considered the global stability of the drug equilibrium under some conditions. However, they treated the total population is a constant for simple. In fact, we sum the tree equations of (1.1) and get

$$
\frac{\mathrm{d} N}{\mathrm{~d} t}=\Lambda-\mu N-\delta_{1} U_{1}-\delta_{2} U_{2} .
$$

It is easy to see that the total population is not a constant. This assumption is not reasonable and maybe simply the problem. Since (1.1) can be changed a plane system under the constant population. In our paper we will relax this assumption and assume the total population is very according to the time. In our model we use the bilinear law incidence function instead of standard incidence. The model is changed into 


$$
\begin{aligned}
& \frac{\mathrm{d} S}{\mathrm{~d} t}=\Lambda-\beta_{1} S U_{1}-\mu S \\
& \frac{\mathrm{d} U_{1}}{\mathrm{~d} t}=\beta_{1} S U_{1}+\beta_{3} U_{1} U_{2}-\left(p+\mu+\delta_{1}\right) U_{1} \\
& \frac{\mathrm{d} U_{2}}{\mathrm{~d} t}=p U_{1}-\beta_{3} U_{1} U_{2}-\left(\mu+\delta_{2}\right) U_{2}
\end{aligned}
$$

\section{Basic Reproduction Number and Stability of Drug-Free Equilibrium}

The drug-free equilibrium is given by

$$
E_{0}=\left(S^{0}, 0,0\right)=\left(\frac{\Lambda}{\mu}, 0,0\right) .
$$

The basic reproduction number $R_{0}$ is defined to be the expected number of secondary cases produced, in a completely susceptible population, by a typical infected individual during its entire period of infection. We follow the recipe of [4] to calculate the basic reproduction number. O. Diekmann et al. in [5] has shown $R_{0}$ as the spectral radius of the next generation matrix. Firstly we need to separate the new infections from other factors. We define $F(X)$ to be the vector which represents the rate of new infections that appear in the population where $X$ is a vector given by $X=\left(U_{1}, U_{2}, S\right)$. In this model, we get $F=\left(\beta_{1} S U_{1}, 0,0\right)$,

$$
\begin{aligned}
V= & \left(-\beta_{3} U_{1} U_{2}+\left(p+\mu+\delta_{1}\right) U_{1}, \beta_{3} U_{1} U_{2}\right. \\
& \left.+\left(\mu+\delta_{2}\right) U_{2}-p U_{1}, \beta_{1} U_{1} S+\mu S-\Lambda\right)
\end{aligned}
$$

$F, V$ have the same definition as explained in [4]. According to the recipe presented in [4] we define the derivatives $D F\left(E_{0}\right)$ and $D V\left(E_{0}\right)$ in the following way

$$
F=\left(\begin{array}{ccc}
\frac{\beta_{1} \Lambda}{\mu} & 0 & 0 \\
0 & 0 & 0 \\
0 & 0 & 0
\end{array}\right)
$$

and

$$
V=\left(\begin{array}{ccc}
p+\mu+\delta_{1} & 0 & 0 \\
-p & \mu+\delta_{2} & 0 \\
\frac{\beta_{1} \Lambda}{\mu} & 0 & \mu
\end{array}\right) .
$$

Finally the basic reproduction number is obtained by calculating the spectral radius of $F V^{-1}$. Then the basic reproduction number for the model presented in system (1.2) is given by the following expression

$$
R_{0}=\rho\left(F V^{-1}\right)=\frac{\beta_{1} \Lambda}{\mu\left(p+\mu+\delta_{1}\right)} .
$$

This completes the calculation of basic reproduction number. The Jacobian matrix of (1.2) at drug-free equilibrium $E_{0}$ is

$$
J_{E_{0}}=\left(\begin{array}{ccc}
-\mu & -\frac{\beta_{1} \Lambda}{\mu} & 0 \\
0 & \frac{\beta_{1} \Lambda}{\mu}-\left(p+\mu+\delta_{1}\right) & 0 \\
0 & p & -\left(\mu+\delta_{2}\right)
\end{array}\right)
$$

The characteristic roots of the matrix are

$$
\lambda_{1}=-\mu<0, \lambda_{2}=-\left(\mu+\delta_{2}\right)<0, \lambda_{3}=\frac{\beta_{1} \Lambda}{\mu}-\left(p+\mu+\delta_{1}\right) .
$$

Hence the characteristic equation just has negative real part roots under some condition. Then we can obtain the following theorem.

Theorem 2.1. If $R_{0}<1$, the drug-free equilibrium is locally asymptotically stable otherwise it is unstable.

Theorem 2.2. If $\frac{\beta_{1} \Lambda}{\mu\left(\mu+\delta_{1}\right)}<1$ drug-free equilibrium is globally stable.

Proof. We set a Lyapunov function $V\left(t, U_{1}, U_{2}\right)=U_{1}+U_{2}$.

Then

$$
\begin{aligned}
\left.\frac{\mathrm{d} V}{\mathrm{~d} t}\right|_{(2.1)} & =U_{1}+U_{2} \\
& =\beta_{1} S U_{1}-\left(\mu+\delta_{1}\right) U_{1}-\left(\mu+\delta_{2}\right) U_{2} \\
& \leq\left(\mu+\delta_{1}\right)\left(\frac{\beta_{1} \Lambda}{\mu\left(\mu+\delta_{1}\right)}-1\right) U_{1}-\left(\mu+\delta_{2}\right) U_{2}
\end{aligned}
$$

When $\frac{\beta_{1} \Lambda}{\mu\left(\mu+\delta_{1}\right)}<1$, it is easy to get $\frac{\mathrm{d} V}{\mathrm{~d} t} \leq 0$. Also $\dot{V}=0$ if and only if $U_{1}, U_{2}$ are all zero. Note that the largest invariant set is $M=\left\{\left(\frac{\Lambda}{\mu}, 0,0\right) \mid \frac{\mathrm{d} V}{\mathrm{~d} t}=0\right\}$, the drug-free equilibrium $E_{0}$ is globally asymptotically stable by Lassale's invariance principle.

\section{Existence and Stability of the Endemic Equilibrium}

In this section we discuss the existence of the endemic equilibrium. We just consider the special situation. We find the positive endemic equilibrium for (1.2) which is obtained by solving the following sets of equations 


$$
\left\{\begin{array}{l}
\Lambda-\beta_{1} S^{*} U_{1}^{*}-\mu S^{*}=0, \\
\beta_{1} S^{*} U_{1}^{*}+\beta_{3} U_{2}^{*} U_{3}^{*}-\left(p+\mu+\delta_{1}\right) U_{1}^{*}=0, \\
p U_{1}^{*}-\beta_{3} U_{1}^{*} U_{2}^{*}-\left(\mu+\delta_{2}\right) U_{2}^{*}=0 .
\end{array}\right.
$$

Solving the first and second equations of (3.1), we obtain

$$
U_{1}^{*}=\frac{\Lambda-\mu S^{*}}{\beta_{1} S^{*}}, U_{2}^{*}=\frac{p+\mu+\delta_{1}}{\beta_{3}} \text {. Substituting } U_{1}^{*} \text { and }
$$

$U_{2}^{*}$ into the third equation of (3.1), we obtain $S^{*}$ should satisfies the following equation

$$
P\left(E^{*}\right)=A x^{2}+B x+C=0 \text {. }
$$

where

$$
\begin{aligned}
& A=\beta_{1}^{2}\left(\mu+\delta_{2}\right)-\beta_{1} \beta_{3} \mu, \\
& B=\beta_{1} \beta_{3} \Lambda+\beta_{3} \mu\left(p+\mu+\delta_{1}\right)-\beta_{1}\left(\mu+\delta_{2}\right)\left(\mu+\delta_{1}\right), \\
& C=-\beta_{3} \Lambda\left(\mu+\delta_{1}\right)
\end{aligned}
$$

In our model, we just consider $\beta_{1}>\beta_{3}$. It is easy to see $A>0$ and $C<0$. Due to Descarters' rule of sings, the number of real positive roots of $P\left(E^{*}\right)$ is determined by the following table

\begin{tabular}{cc}
\hline B & Number of Real Positive Roots \\
\hline$>0$ & 1 \\
$<0$ & 1 \\
\hline
\end{tabular}

From the expression of $U_{1}^{*}$ and $U_{2}^{*}$, and positivity of the endemic equilibrium, we note that

$$
S^{*}<\min \left\{\frac{\Lambda}{\mu}, \frac{p+\mu+\delta_{1}}{\beta_{1}}\right\}
$$

If $R_{0}>1, S^{*}<\frac{p+\mu+\delta_{1}}{\beta_{1}}$. We assume the two real positive roots of (1.2) are respectively $x_{-}$and $x_{+}$, we check the positive real roots of (1.2)

\begin{tabular}{cc}
\hline$x$ & Number of Real Positive Roots of (3.1) \\
\hline$x_{+} \leq \frac{p+\mu+\delta_{1}}{\beta_{1}}$ & 1 \\
$x_{+}>\frac{p+\mu+\delta_{1}}{\beta_{1}}$ & 0 \\
\hline
\end{tabular}

Concluding the above discussion, then we can obtain the following theorem.

Theorem 3.1. 1) If $R_{0}>1$, and $x_{+} \leq \frac{p+\mu+\delta_{1}}{\beta_{1}}$,

has only unique real positive endemic equilibrium.

Next, we will discuss the locally stability of the endemic equilibrium. The Jacobian matrix at endemic equilibrium is

$$
J_{E^{*}}=\left(\begin{array}{ccc}
-\beta_{1} U_{1}^{*}-\mu & -\beta_{1} S^{*} & 0 \\
\beta_{1} U_{1}^{*} & \beta_{1} S^{*}+\beta_{3} U_{2}^{*}-\left(p+\mu+\delta_{1}\right) & \beta_{3} U_{1}^{*} \\
0 & p-\beta_{3} U_{2}^{*} & -\beta_{3} U_{1}^{*}-\left(\mu+\delta_{2}\right)
\end{array}\right) .
$$

Then the characteristic of the endemic equilibrium is

$$
\lambda^{3}+a_{1} \lambda^{2}+a_{2} \lambda+a_{3}=0,
$$

where

$$
\begin{aligned}
& a_{1}=\beta_{3} U_{1}^{*}+2 \mu+\delta_{1}+\beta_{1} U_{1}^{*}>0 \\
& a_{2}=\left(\beta_{1} U_{1}^{*}+\mu\right)\left(\beta_{3} U_{1}^{*}+\mu+\delta_{2}\right)+\beta_{3} U_{1}^{*}\left(\mu+\delta_{1}\right)+\beta_{1}\left(\beta_{1}-\beta_{3}\right) S^{*} U_{1}^{*}>0, \\
& a_{3}=\beta_{3} U_{1}^{*}\left(\beta_{1} U_{1}^{*}+\mu\right)\left(\mu+\delta_{1}\right)+\beta_{1}\left(\beta_{1}-\beta_{3}\right) \mu S^{*} U_{1}^{*}+\beta_{1}^{2} S^{*} U_{1}^{*}\left(\mu+\delta_{2}\right)>0 .
\end{aligned}
$$

Define $H\left(E^{*}\right)=a_{1} a_{2}-a_{3}$, and straightly calculate and then we obtain

$$
H\left(E^{*}\right)=\left(\beta_{3} U_{1}^{*}+\mu+\delta_{2}\right)\left[\left(\beta_{1} U_{1}^{*}+\mu\right)^{2}+\left(\beta_{3} U_{1}^{*}+\mu+\delta_{2}\right)\left(\beta_{1} U_{1}^{*}+\mu\right)-\left(\mu+\delta_{2}\right) \beta_{3} U_{2}^{*}\right]+\beta_{1}^{2} S^{*} U_{1}^{*}\left(\beta_{1} U_{1}^{*}+\mu\right) .
$$

Define $y=\left(\beta_{1} U_{1}^{*}+\mu\right)$, and $f(y)=y^{2}+\left(\beta_{3} U_{1}^{*}+\mu+\delta_{2}\right)-\left(\mu+\delta_{2}\right) \beta_{3} U_{2}^{*}$. Note that $\Delta=\left(\beta_{3} U_{1}^{*}+\mu+\delta_{2}\right)^{2}+4\left(\mu+\delta_{2}\right) \beta_{3} U_{2}^{*}>0$, it is easy to get $H\left(E^{*}\right)>0$. Due to the Routh Hurtwiz [6], we get the following theorem.
Theorem 3.2. Let $E^{*}$ be the endemic equilibria of system (1.2) as defined before, and assume $x_{+} \leq \frac{p+\mu+\delta_{1}}{\beta_{1}}$ and $R_{0}>1$, then it is locally asymptotically stable. 
Finally, we will consider the global stability of the endemic equilibrium is asymptotically orbitally stable with asymptotic phase.

Theorem 3.3. The endemic equilibrium of $E^{*}$ to
(1.2), if it exists, is asymptotically orbitally stable with asymptotic phase.

Proof. The second compound matrices $J\left(S, U_{1}, U_{2}\right)$ of (1.2) is given by

$$
\begin{aligned}
& \left(\begin{array}{c}
-\beta_{1} U_{1}+\beta_{1} S+\beta_{3} U_{2} \\
-\left(p+2 \mu+\delta_{1}\right) \\
p-\beta_{3} U_{2} \\
0
\end{array}\right)-\beta_{1} U_{1}-\beta_{3} U_{1} \\
& \text { we can write the linear system (1.2) with respect to a } \\
& \text { solution }\left(S(t), U_{1}(t), U_{2}(t)\right) \text { of }(1.2) \text { as the following } \\
& 3 \times 3 \text { system }(3.4): \\
& X^{\prime}=-\left(\beta_{1} U_{1}+p+2 \mu+\delta_{1}-\beta_{1} S-\beta_{3} U_{2}\right) X+\beta_{3} U_{1} Y, \\
& Y^{\prime}=\left(p-\beta_{3} U_{2}\right) X-\left[\left(\beta_{1}+\beta_{3}\right) U_{1}+2 \mu+\delta_{2}\right] Y-\beta_{1} S Z, \\
& Z=\beta_{1} U_{1} Y-\left[p+2 \mu+\delta_{1}+\delta_{2}-\beta_{1} S-\beta_{3} U_{2}+\beta_{3} U_{1}\right) Z .
\end{aligned}
$$

To show the asymptotic stability of the system (3.4)

we consider the following function:

$$
V\left(X, Y, Z ; S, U_{1}, U_{2}\right)=\left|P\left(S, U_{1}, U_{2}\right) \cdot(X, Y, Z)^{*}\right|
$$

where the matrix $P=\operatorname{diag}\left(I, U_{1} / U_{2}, U_{1} / U_{2}\right)$ and $\mid \cdot$ is the norm in $R^{3}$ defined by

$$
D_{+}|X(t)| \leq-\left(\beta_{1} U_{1}+p+2 \mu+\delta_{1}-\beta_{1} S-\beta_{3} U_{2}\right)|X|+\beta_{3} U_{1}\left(\frac{U_{1}}{U_{2}}(|Y|+|z|)\right),
$$

and

$$
\begin{aligned}
& D_{+}|Y| \leq\left(p-\beta_{3} U_{2}\right)|X|-\left[\left(\beta_{1}+\beta_{3}\right) U_{1}+2 \mu+\delta_{2}\right]|Y|-\beta_{1} S|Z| \\
& D_{+}|Z| \leq \beta_{1} U_{1}|Y|-\left[p+2 \mu+\delta_{1}+\delta_{2}-\beta_{1} S-\beta_{3} U_{2}+\beta_{3} U_{1}\right)|Z|
\end{aligned}
$$

and thus

$$
\begin{aligned}
& D_{+} \frac{U_{1}}{U_{2}}(|Y|+|z|)=\left(\frac{U_{1}^{\prime}}{U_{1}}-\frac{U_{2}^{\prime}}{U_{2}}\right) \frac{U_{1}}{U_{2}}(|Y|+|z|)+\frac{U_{1}}{U_{2}} D_{+}(|Y|+|z|), \\
& \leq \frac{U_{1}}{U_{2}}\left(p-\beta_{3} U_{2}\right)|X|+\frac{U_{1}}{U_{2}}(|Y|+|z|)\left(\frac{U_{1}^{\prime}{ }_{1}}{U_{1}}-\frac{U^{\prime}{ }_{2}}{U_{2}}-\beta_{3} U_{1}-2 \mu-\delta_{2}+\beta_{3} U_{2}\right)(|Y|+|z|) .
\end{aligned}
$$

We claim that

$$
D_{+} V(t) \leq \sup \left\{g_{1}(t), g_{2}(t) V(t)\right\}
$$

where

$$
\begin{aligned}
& g_{1}(t)=-\beta_{1} U_{1}-p-2 \mu-\delta_{1}+\beta_{1} S, \\
& g_{2}(t)=\frac{U^{\prime}{ }_{1}}{U_{1}}-\frac{U^{\prime}{ }_{2}}{U_{2}}-\beta_{3} U_{1}-2 \mu-\delta_{2}+\beta_{3} U_{2}
\end{aligned}
$$

Using (1.2), we find

$$
g_{1}(t)=\frac{U^{\prime}{ }_{1}}{U_{1}}-\beta_{1} U_{1}-\beta_{3} U_{2}-\mu \leq \frac{U^{\prime}{ }_{1}}{U_{1}}-\mu
$$

and

$$
g_{2}(t)=-\frac{p U_{1}}{U_{2}}<0
$$

and thus

$$
\int_{0}^{w} \sup \left\{g_{1}(t), g_{2}(t)\right\} \mathrm{d} t \leq\left.\log U_{1}(t)\right|_{0} ^{w}-\mu w<0
$$


which, together with (3.9), implies that $V(t) \rightarrow 0$ as $t \rightarrow \infty$ and in turn that $(X(t), Y(t), Z(t)) \rightarrow 0$ as $t \rightarrow \infty$ by (3.6). As a result, the linear system (1.2) is asymptotically stable and the periodic solution $\left(S(t), U_{1}(t), U_{2}(t)\right)$ is asymptotically orbitally stable with asymptotic phase by Theorem 3.1 [7].

\section{Conclusions and Simulation}

In this paper we have addressed the problem of investigation the existence and stability of the equilibria heroin epidemic model provided by Mulone and Straughan. We relax the condition the total population is a constant. Under some condition, the drug-free equilibrium is global asymptotically stable; the number of equilibria are determined by Theorem 3.1 and we show that the endemic equilibrium is also globally asymptotically stable under any conditions. The natures of the model are not different from the epidemic models $[1,3]$. We use numerical simulations to illustrate the theoretical results obtained in previous sections. As an example, we take the parameter values as follows: $\Lambda=1, \beta_{1}=0.008$, $\beta_{3}=0.001, p=0.04, \mu=0.01, \delta_{1}=0.05, \delta_{2}=0.06$ Ву using the classical implicit format solving the ordinary differential equations and the method of steps for differential equations, we can solve the numerical solutions of (1.2) via the software package Matlab (see Figure 1) is globally asymptotically stable with asymptotic phase. The initial conditions are $S_{0}=10, U_{10}=1, U_{20}=1$.

In future, there are some problems that will be solved. We continue the problems of system (2.1) on substituting the bilinear into standard incidence. Whether there are some kinds of bifurcations for the model or not is still open. If we assume the input is impulsive input or sto-

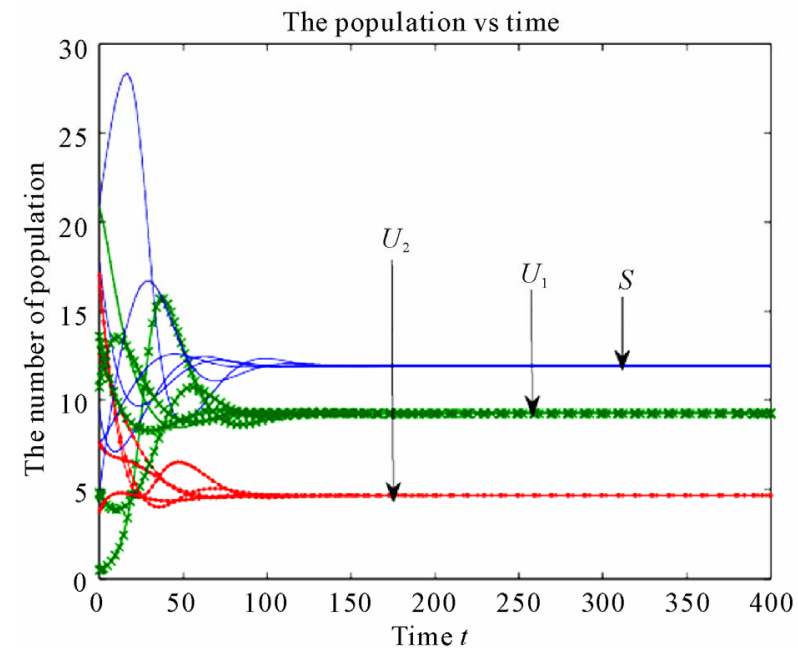

(a) chastic perturbations, what results will occur. We give some numerical results. For the impulsive perturbation, we give the following system (4.1).

$$
\left\{\begin{array}{l}
\frac{\mathrm{d} S}{\mathrm{~d} t}=-\beta_{1} S U_{1}-\mu S, t \neq n T, \\
S\left(t^{+}\right)=S(t)+\Lambda, t=n T, \\
\frac{\mathrm{d} U_{1}}{\mathrm{~d} t}=\beta_{1} S U_{1}+\beta_{3} U_{1} U_{2}-\left(p+\mu+\delta_{1}\right) U_{1}, t \neq n T, \\
U_{1}\left(t^{+}\right)=U_{1}(t), t=n T, \\
\frac{\mathrm{d} U_{2}}{\mathrm{~d} t}=p U_{1}-\beta_{3} U_{1} U_{2}-\left(\mu+\delta_{2}\right) U_{2}, t \neq n T, \\
U_{2}\left(t^{+}\right)=U_{2}(t), t=n T .
\end{array}\right.
$$

Theorem 4.1. The drug-free periodic solution of (4.1) is globally asymptotically stable, and the system is persistent.

As another example, we take the parameter values as follows: $\Lambda=2, \beta_{1}=1, \beta_{3}=0.1, p=0.4, \mu=0.01$, $\delta_{1}=0.05, \delta_{2}=0.06, T=1$. We can solve the numerical solutions of (4.1) via the software package Matlab (see Figure 2).

For the stochastic perturbation, we give the following system (4.2).

$$
\left\{\begin{array}{l}
\frac{\mathrm{d} S}{\mathrm{~d} t}=\Lambda-\beta_{1} S U_{1}-\mu S+\sigma \mathrm{d} \xi_{t}^{1}, \\
\frac{\mathrm{d} U_{1}}{\mathrm{~d} t}=\beta_{1} S U_{1}+\beta_{3} U_{1} U_{2}-\left(p+\mu+\delta_{1}\right) U_{1}+\sigma \mathrm{d} \xi_{t}^{2}, \\
\frac{\mathrm{d} U_{2}}{\mathrm{~d} t}=p U_{1}-\beta_{3} U_{1} U_{2}-\left(\mu+\delta_{2}\right) U_{2}+\sigma \mathrm{d} \xi_{t}^{3} .
\end{array}\right.
$$

Phase portrait

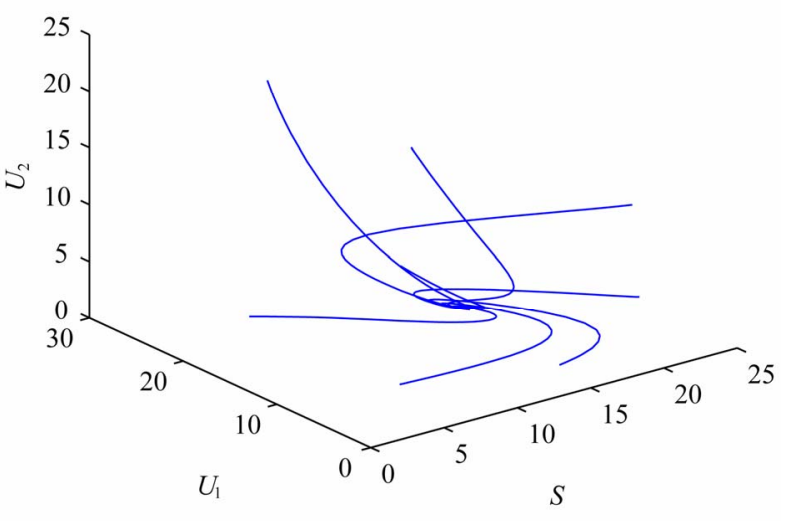

(b)

Figure 1. (a)-(b) show that endemic equilibrium to (1.2). 


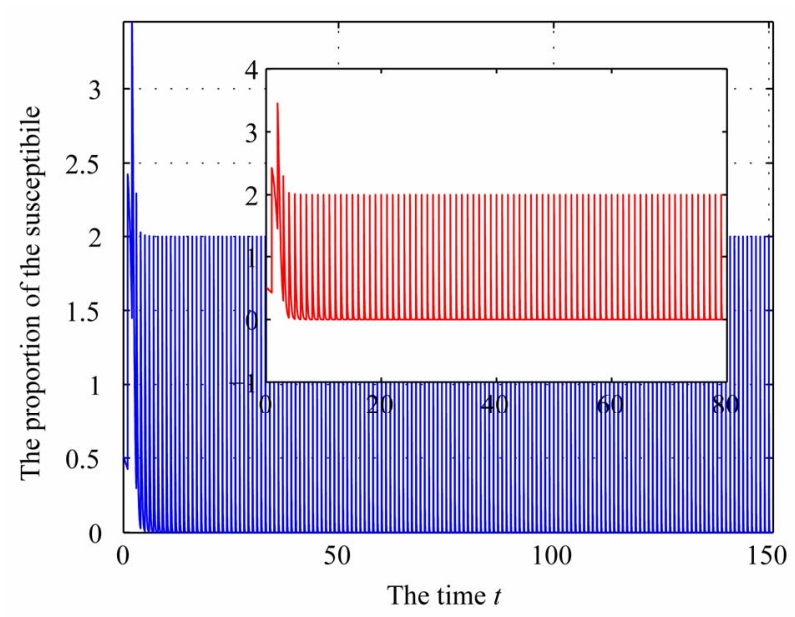

(a)

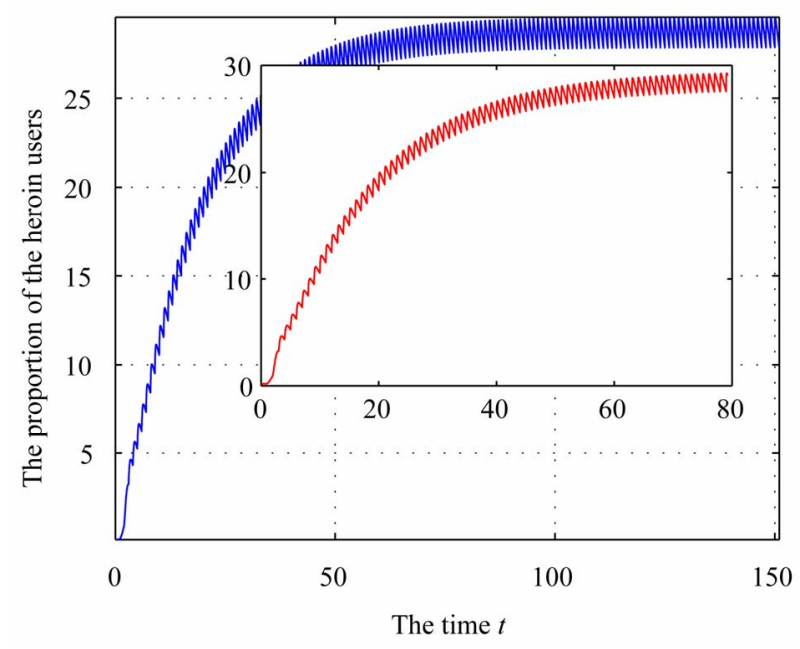

(b)

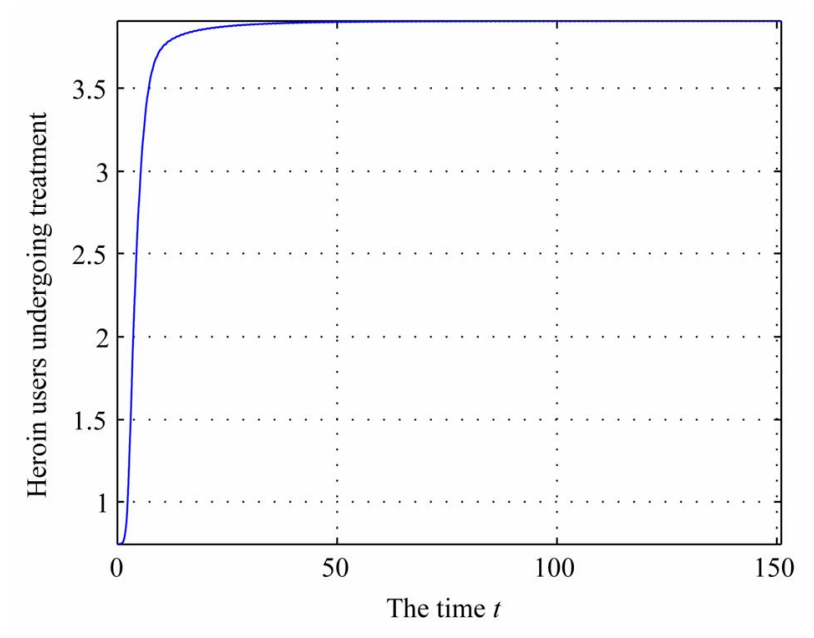

(c)

Figure 2. (a)-(c) show that the system (4.1) is persistent. The initial conditions are $S_{0}=0.5126, U_{10}=0.1688, U_{20}=0.7448$.

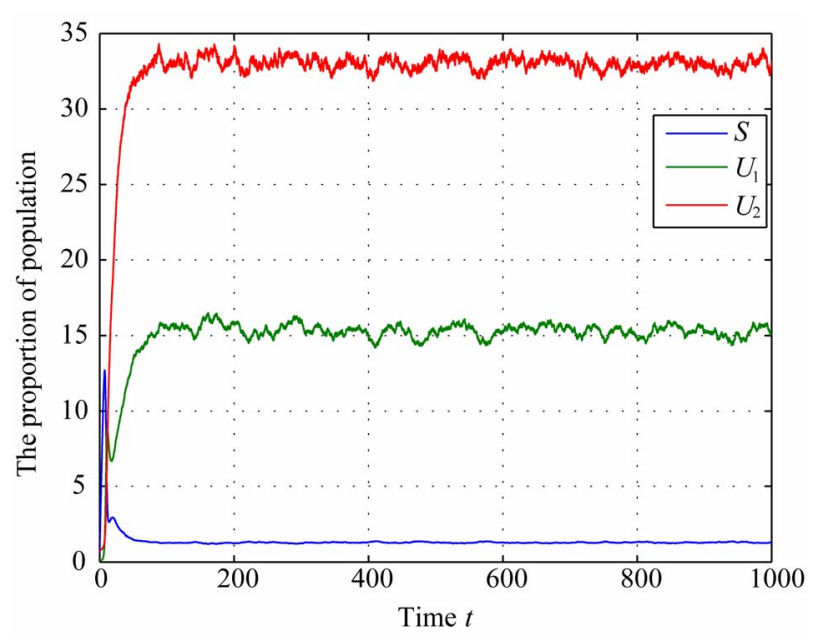

(a)

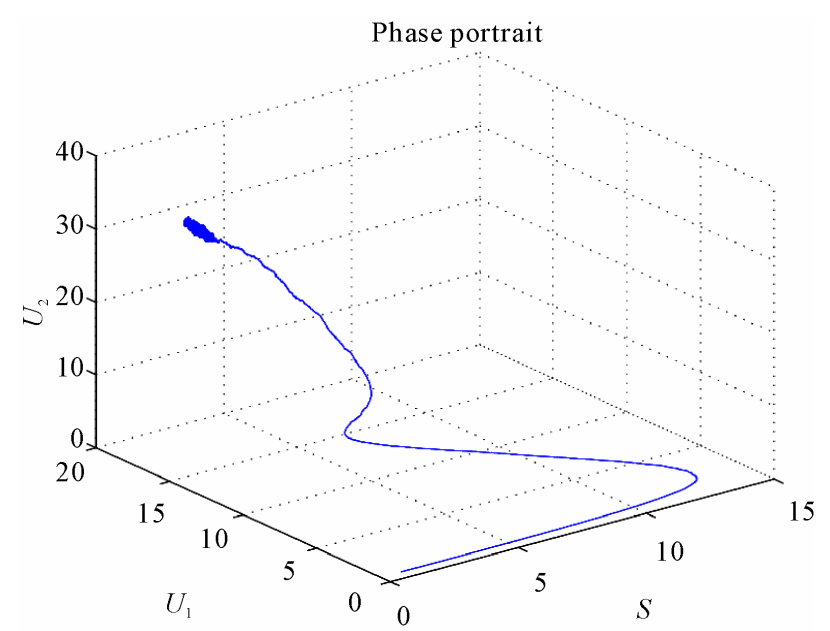

(b)

Figure 3. (a)-(b) show that the zero solution of system (4.2) is probably asymptotically stable. The initial conditions are $S_{0}=0.5126, U_{10}=0.1688, U_{20}=0.7448$.

We take the parameter values as follows: $\Lambda=2$,

$$
\begin{aligned}
& \beta_{1}=0.1, \beta_{3}=0.01, p=0.4, \mu=0.01, \\
& \delta_{1}=0.05, \delta_{2}=0.06, \sigma=0.002 .
\end{aligned}
$$

we can solve the numerical solutions of (4.1) via the software package Matlab (see Figure 3).

Furthermore, we will give the detail analysis proofs for the theorems in the future.

\section{Acknowledgements}

We thank the anonymous referee for his (or her) aluable comments and suggestions on the previous version of this paper. 


\section{References}

[1] G. Mulone and B. Straughan, "A Note on Heroin Epidemics," Mathematical Biosciences, Vol. 218, No. 2, 2009, pp. 138-141. doi:10.1016/j.mbs.2009.01.006

[2] NIDA InfoFacts: Heroin. http://www.nida.nih.gov/infofacts/heroin.html

[3] E. White and C. Comiskey, "Heroin Epidemics, Treatment and ODE Modelling," Mathematical Biosciences, Vol. 208, No. 1, 2007, pp. 312-324. doi:10.1016/j.mbs.2006.10.008

[4] P. Driessche and W. James, "Reproduction Numbers and Subthreshold Endemic Eqilibria for Compartmental Models of Disease Transmission," Mathematical Biosciences,
Vol. 180, No. 1-2, 2002, pp. 29-48. doi:10.1016/S0025-5564(02)00108-6

[5] O. Diekmann, J. A. P. Heesterbeek and J. A. J. Metz, "On the Definition and the Computation of the Basic Reproduction Ratio $R_{0}$ in Models for Infectious Diseases in Heterogenous Populations," Journal of Mathematical Biology, Vol. 28, No. 4, 1990, pp. 365-382. doi:10.1007/BF00178324

[6] M. Y. Li and J. S. Muldowney, "Global Stability for the SEIR Model in Epidemiology," Mathematical Biosciences, Vol. 125, No. 2, 1995, pp. 155-164. doi:10.1016/0025-5564(95)92756-5

[7] J. K. Hale, "Ordinary Differential Equations," John Wiley and Sons, New York, 1969. 